\title{
YABALÁ: módulo de software para asistir en el uso de licencias Creative Commons en REA
}

\author{
José Fager ${ }^{1}$, Regina Motz ${ }^{2}$ \\ ${ }^{1}$ Facultad de Información y Comunicación - Universidad de la República (UdelaR) \\ Montevideo - Uruguay \\ ${ }^{2}$ Facultad de Ingeniería - Universidad de la República (UdelaR) \\ Montevideo - Uruguay \\ jose.fagerefic.edu.uy, rmotzefing.edu.uy
}

\begin{abstract}
Resumen. Este artículo describe el proceso de desarrollo de un módulo de software "Open Source" (OS) denominado Yabalá, el que tiene como objetivo permitir la implementación de aplicaciones que puedan brindar funcionalidades como: cálculo de licencias de recursos, derivados de la combinación de otros que están bajo licencia "Creative Commons" (CC), despliegue de los créditos y licencia de dichos recursos según los requerimientos de la versión 4.0 de CC. Se detalla el uso del módulo para el desarrollo de una aplicación de software específica (Calculator) y la forma en que es usada en Moodle, Moodle Hub, WordPress, Joomla y Drupal.
\end{abstract}

\section{El problema de la combinación de obras con licencia $\mathrm{CC}$}

Está prevista la posibilidad de reutilizar obras que poseen una licencia CC, existen dos posibles situaciones, la modificación de una obra ("obra adaptada") y la combinación de dos o más obras ("remix"), en ambos casos la obra resultante es llama "obra derivada".

Al realizar un remix de obras no siempre las licencias de las obras a remixar son compatibles de manera de poder ser combinadas para derivar una nueva obra a partir de ellas. Las compatibilidades entre diferentes licencias con el objeto de ser usadas en remixes se pueden ver en la "Tabla de compatibilidad de licencias"1.

También lo anterior trae aparejado la necesidad de hacer una correcta atribución en los créditos de las obras utilizadas según las "Buenas prácticas para la atribución", las que sin obligar sugieren formas de atribuir las obras reutilizadas.

\section{Desarrollo de Yabalá}

Yabalá es un módulo desarrollado en leguaje de programación PHP, código abierto y liberado bajo licencia GPL3. Se presenta como una interfaz que ofrece un conjunto de funciones y procedimientos, pensados para ser usados en el desarrollo de aplicaciones que implementen herramientas $\mathrm{CC}$, sus principales funcionalidades son:

\footnotetext{
${ }^{1}$ https://wiki.creativecommons.org/wiki/Wiki/cc_license_compatibility

${ }^{2} \mathrm{https}$ ///wiki.creativecommons.org/wiki/Best_practices_for_attribution
} 
- Retornar una lista de repositorios donde se puedan hacer búsquedas de obras que poseen distintos tipos y formas de licenciamiento (con la intención permitir implementar sistemas de búsquedas federadas).

- Hacer una búsqueda de obras en un repositorio determinado.

- Agregar una obra al sistema indicando datos básicos de la misma y su licencia.

- Retornar las posibles licencias que pudiera tener una obra derivada producto de la combinación o reutilización de todas las obras cargadas al momento en el sistema.

- Retornar la licencia más restrictiva que pudiera tener una obra derivada producto de la combinación o reutilización de todas las obras cargadas al momento en el sistema.

- Retornar la licencia menos restrictiva que pudiera tener una obra derivada producto de la combinación o reutilización de todas las obras cargadas al momento en el sistema.

- Brindar diferentes formas de presentar créditos de una obra derivada, retorna una URL con la información de los créditos alojada en forma permanente en la web la cual puede ser referenciada a futuro, una imagen QR con la información de los créditos, una imagen QR con la licencia de la obra, y la imagen estándar de la licencia de la obra.

\section{Yabalá ejemplo de uso: "Calculator"}

Una de las principales aplicaciones de Yabalá es permitir crear herramientas CC, un ejemplo de esto es la aplicación "Calculator" la cual utiliza el módulo Yabalá, brindando al usuario la posibilidad de calcular una licencia de una recurso derivado de la combinación de otros recursos y los créditos del recurso derivado. Un prototipo funcional de "Calculator" está disponible en: http://164.73.15.7/yabala/calculator/.

"Calculator" es una aplicación que permite a los usuarios administrar "conjuntos de materiales" (por "materiales" se puede entender obras o recursos) sindicado datos básicos de los mismos, sus operaciones elementales son:

- crear un conjunto de materiales,

- agregar una material a un conjunto de materiales,

- quitar un material del conjunto de materiales.

Haciendo un paralelismo entre el concepto de "conjunto de materiales" y "obra derivada", tomando en cuenta a la "obra derivada" como el resultante de combinar todos los materiales disponibles en el conjunto, se puede decir que "Calculator" permite:

- Determinar si es compatible o no combinar el conjunto de materiales en una misma obra derivada. 


\section{CBIE-LACLO 2015}

Anais dos Workshops do IV Congresso Brasileiro de Informática na Educação (CBIE 2015)

- En caso de cumplir lo anterior, ofrecer todas las posibles licencias que podría llegar a tener la obra derivada.

- De las posibles licencias indicar al usuario cual es la más y la menos restrictiva.

- Permitir al usuario elegir una licencia (de las posibles) para la obra derivada.

- Crear una web de créditos de la obra derivada incluyendo la licencia seleccionada por el usuario, generando cuatro URLs permanentes (que se le ofrecen al usuario) para que las pueda referenciar de donde considere necesario, una URL apunta a los créditos en formato HTML, otra a los créditos en formato QR (una imagen PNG), la tercera a un mini QR que solamente indica la licencia de la obra y la cuarta a una imagen tradicional con la licencia de la obra (imagen PNG).

Como ejemplo de uso de "Calculator" podemos suponer tenemos los siguientes cuatro recursos:

- Obra 1: Una imagen con licencia CC0, cuyo autor es “攝到掛” y está ubicada en la dirección "https://www.flickr.com/photos/md903152/19421411035/in/photostream/".

- Obra 2: Un video con licencia BY, cuyo autor es "Kali Group travel" y está ubicada en la dirección "https://www.youtube.com/watch?v=oI2Yj7fzf84".

- Obra 3: Una imagen con licencia PD, cuyo autor es "Jefftopham" y está ubicada en la dirección "https://commons.wikimedia.org/wiki/File:Mummy_Mountain.JPG”.

- Obra 4: Una audio con licencia BY-NC, cuyo autor es "Forssto" y está ubicada en la dirección "https://www.jamendo.com/es/track/1044250/positive-pop-track".

La inclusión de estos cuatro recursos en un conjunto permitirá a "Calculator" indicar:

- si es compatible la combinación de estos recursos en una misma obra derivada;

- en caso de ser compatibles las posible licencia que la obra derivada podría tener;

- y finalmente la opción de crear los links permanentes a los créditos con la licencia seleccionada en formato HTML y QR, así como a la licencia seleccionada en formato QR y tradicional.

Una vez ingresados los datos de las obras y elegida una de las posibles licencias (en el ejemplo se elige BY-NC), se obtienen los siguientes productos:

- Link a los créditos (HTML) http://164.73.15.7/yabala/yabala/www/ejemplo.html.

- Link a los créditos (QR) http://164.73.15.7/yabala/yabala/www/ejemplo full.png.

- Link a la licencia (tradicional) http://164.73.15.7/yabala/yabala/www/ejemplo_cc.png.

- Link la licencia (QR) http://164.73.15.7/yabala/yabala/www/ejemplo_min.png. 


\section{Uso de los productos de "Calculator" en LMS y CMS}

Lo lógico es que el usuario quiera usar los productos obtenidos con "Calculator" para indicar los créditos y las licencias de la obra derivada (por ejemplo, una página web en WordPress o un curso en Moodle), por eso a continuación se muestra cómo usar dichos productos en Moodle, Moodle Hub, Joomla y Drupal respectivamente.

\section{Moodle}

Una situación que se puede dar es que un curso el docente tenga varios recursos disponibles y que cada recurso tenga una licencia respectiva, la combinación de esas licencias va a determinar la posible licencia que el curso completo pueda tener, ahora que licencia, que créditos y como ponerlos en disponibilidad son tareas que "Calculator" puede resolver. Tomando en cuenta el ejemplo de los cuatro recursos vistos en este trabajo, se puede insertar en un curso cualquiera de los elementos que se obtuvieron en el ejemplo anterior; insertándolos en por ejemplo una "etiqueta" de un curso, un "bloque HTML", o cualquier otro recurso del curso que permita insertar hipervínculos o imágenes. Se puede ver el ejemplo citado en un curso de Moodle ingresando en la siguiente dirección: http://eva.universidad.edu.uy/course/view.php?id=4111.

\section{Moodle Hub}

Una situación similar se puede presentar al definir la licencia de un curso que se indexa en un repositorio de cursos implementado con Moodle Hub, al momento de querer remitir un curso al repositorio se puede indicar su licencia usando los productos obtenidos con "Calculator". Se puede ver el ejemplo citado como un curso Moodle que fue remitido a un repositorio de cursos en la siguiente dirección: http://cursosabiertos.edu.uy/?courseid=22.

\section{WordPress, Joomla y Drupal}

En forma similar al ejemplo visto en Moodle, se puede usar los productos de "Calculator" en aplicaciones CMS, se puede acceder a los ejemplos en la web del uso de "Calculator" en:

- WordPress: http://164.73.15.7/wordpress.html

- Joomla: http://164.73.15.7/joomla.html

- Drupal: http://164.73.15.7/drupal.html

\section{Limitaciones, ventajas y trabajo a futuro} puntualizar.

El estado actual de la aplicación, trae algunas limitaciones que es importante

Ingreso de datos: la recopilación de la información (licencias, título, url, etc.) de los recurso a usar, debe ser proporcionado por un usuario humano en forma "manual", sería deseable que en los casos que los recursos a utilizar que estén disponibles en repositorios como Flickr o Youtube (por ejemplo), se pueda obtener dicha información en forma directa y dar eventualmente la opción de su modificación al usuario.

Integración con CMS y LMS: En la actualidad la integración se realiza por medio de la inyección de código a través de las funcionalidades que las aplicaciones ya 
tienen disponibles, es decir de manera "manual" debe embeberse la referencia ya sea a la licencia o a los créditos de la obra derivada, es deseable (y uno de los objetivos) que aplicaciones como Moodle y WordPress (por ejemplo) usen Yabalá en forma nativa e implemente funcionalidades que permitan automáticamente insertar la licencia y generar los créditos del contenido que administran (por ejemplo un artículo en una web en el caso de WordPress o en un curso en el caso de Moodle).

Pero al mismo tiempo posee algunas ventajas que colocarían al desarrollo actual en condiciones de poder subsanar a futuro las limitaciones anteriormente mencionadas.

Yabalá es código abierto, está disponible bajo licencia GPL3, y es posible descargar el código desde Github, así como sus actualizaciones y mejoras. También cuenta con un diseño y desarrollo realizado usando el paradigma de la programación orientada a objetos, teniendo como modelo de diseño el patrón "Facade" lo cual implica que:

- está disponible para ser usada en desarrollos independientes de la línea de desarrollo principal,

- cuenta con las condiciones para poder enriquecerse del aporte de otros desarrollos y desarrolladores,

- está desarrollado en lenguaje PHP que es el mismo que usan Moodle, MoodleHub, Joomla y WordPress,

- su diseño usando el patrón "Facade" tiene la ventaja que pueden ser modificadas las clases del sistema, haciendo que los clientes (aplicaciones que usan Yabalá) pueden permanecer ajenos a dichas modificaciones, esto asegura en buena medida la compatibilidad a futuro de desarrollos que usen el módulo y aumenta su posibilidad de integración en otros desarrollos.

Por lo tanto como principales línea de trabajo a futuro se plantea la mejora paulatina de las limitaciones indicadas. El nivel de integración de Yabalá para ser usado en CMS y LMS no es fuerte ya que implica la inyección manual de la referencia a los productos generados por el módulo, pero si es posible aumentar dicho nivel de integración por el lenguaje de programación usado, el diseño, y la forma de disponibilidad del código para su reutilización. Por las mismas razones es esperable que a futuro la recopilación de información pueda mejorar.

\section{Más información sobre Yabalá}

- Web del proyecto Yabalá: http://164.73.15.7/yabala.

- Código de Yabalá en Github: https://github.com/Yabala/yabala.

- Calculator (aplicación de Yabalá): http://164.73.15.7/yabala/web/calculator.html.

- Simulator (aplicación de Yabalá): http://164.73.15.7/yabala/web/simulator.html.

- Search (aplicación de Yabalá): http://164.73.15.7/yabala/web/search.html. 